\title{
The extreme T Tauri star RW Aur: accretion and outflow variability ${ }^{\star}$
}

\author{
S. H. P. Alencar ${ }^{1, \star \star}$, G. Basri ${ }^{2}$, L. Hartmann ${ }^{3}$, and N. Calvet ${ }^{3}$ \\ 1 Departamento de Física, ICEx-UFMG, CP 702, Belo Horizonte, MG 30123-970, Brazil \\ e-mail: silvia@fisica.ufmg.br \\ 2 Astronomy Department, University of California, Berkeley, CA 94720, USA \\ e-mail: basri@astro.berkeley.edu \\ 3 Harvard-Smithsonian Center for Astrophysics, Cambridge, MA 02138, USA \\ e-mail: [hartmann; ncalvet]@cfa.harvard.edu
}

Received 26 April 2005 / Accepted 7 June 2005

\begin{abstract}
We present an analysis of the classical T Tauri star RW Aur A, based on 77 echelle spectra obtained at Lick Observatory over a decade of observations. RW Aur, which has a higher than average mass accretion rate among T Tauri stars, exhibits permitted $(\mathrm{H} \alpha, \mathrm{H} \beta, \mathrm{Ca} \mathrm{II}, \mathrm{He} \mathrm{I}, \mathrm{NaD})$ and forbidden ([OI]6300 $\AA$ ) emission lines with strong variability. The permitted lines display multiple periodicities over the years, often with variable accretion (redshifted) and outflow (blueshifted) absorption components, implying that both processes are active and changing in this system. The broad components of the different emission lines exhibit correlated behavior, indicating a common origin for all of them. We compute simple magnetospheric accretion and disk-wind $\mathrm{H} \alpha, \mathrm{H} \beta$ and $\mathrm{NaD}$ line profiles for RW Aur. The observed Balmer emission lines do not have magnetospheric accretion line profiles. Our modeling indicates that the wind contribution to these line profiles is very important and must be taken into account. Our results indicate that the $\mathrm{H} \alpha, \mathrm{H} \beta$ and $\mathrm{NaD}$ observed line profiles of RW Aur are better reproduced by collimated disk-winds starting from a small region near the disk inner radius. Calculations were performed in a region extending out to $100 R_{\star}$. Within this volume, extended winds originating over many stellar radii along the disk are not able to reproduce the three lines simultaneously. Strongly open-angled winds also generate profiles that do not look like the observed ones. We also see evidence that the outflow process is highly dynamic - the low- and high-velocity components of the [OI] $(6300 \AA)$ line vary independently on timescales of days. The apparent disappearance from December 1999 to December 2000 of the $[\mathrm{OI}](6300 \AA)$ low velocity component, which is thought to come from the disk-wind, shows that the the slow wind can exhibit dramatic variability on timescales of months (placing limits on how extended it can be). There is no comprehensive explanation yet for the behavior of RW Aur, which may in part be due to complications that would be introduced if it is actually a close binary.
\end{abstract}

Key words. line: profiles - stars: formation - stars: pre-main sequence - stars: winds, outflows - stars: individual: RW Aur

\section{Introduction}

Classical T Tauri stars (CTTSs) are optically visible low-mass Pre-Main Sequence stars surrounded by an accretion disk. They exhibit IR excesses mostly associated with the reprocessing of stellar or accretion radiation by the disk. They also show a flux excess relative to the photosphere in the optical (known as veiling) and in the UV produced in hot spots at the stellar surface, where the infalling material hits the star in the accretion process (Bertout et al. 1988; Calvet \& Gullbring 1998). CTTSs present

^ The reduced spectra corresponding to the observations listed in Table 1 are available in FITS format at CDS via anonymous ftp to cdsarc.u-strasbg.fr $(130.79 .128 .5)$ or via http://cdsweb.u-strasbg.fr/cgi-bin/qcat?J/A+A/440/595

$\star \star$ Present address: Laboratoire d'Astrophysique de Grenoble, Université Joseph-Fourier, BP 53, 38041 Grenoble Cedex 9, France. many permitted and forbidden emission lines and show lithium $(6707.8 \AA)$ in absorption. They are photometrically variable, mainly due to the presence of hot and cold spots at their surface. The cold spots are due to magnetic activity that is also responsible for flares and X-ray emission.

Many decades ago outflowing wind models were suggested to explain the CTTSs' emission line spectra (Kuhi 1964; Hartmann et al. 1982, 1990; Natta \& Giovanardi 1990). The early wind models tended to predict P Cygni profiles (i.e. redshifted emission peaks and blueshifted absorption often going below the continuum). This is not what was typically seen - instead the blue-shifted features usually did not reach the continuum (Bertout 1989). Nonetheless, it was always clear that they represented outflow features, and exhibited different variability than other parts of the line profile (Johns \& Basri 1995a). Wind models were later replaced by magnetospheric accretion 
models (Shu et al. 1994; Hartmann et al. 1994; Muzerolle et al. 1998, 2001) that are the current consensus to describe CTTSs. These models did not attempt to explain the blueshifted absorption features. They invoke a strong stellar magnetic field to truncate the circumstellar disk near the co-rotation radius and lock the star to the disk. Such fields are indeed observed on PMS stars (Basri et al. 1992; Valenti \& Johns-Krull 2004). Some material is accreted through closed magnetic field lines from the disk to the star, while angular momentum is transferred from the star to the disk. Open field lines originating close to the co-rotation radius drive away a wind, and may spiral up to create a jet. Most of the permitted emission lines are produced in the magnetic funnel flow (Hartmann et al. 1994; Muzerolle et al. 2001), while the forbidden emission lines originate in the low density outflow or jet (Shang et al. 2002; Pesenti et al. 2003). When the accretion material hits the stellar surface, a strong shock creates hot spots and the strong continuum excess is produced (Valenti et al. 1993; Calvet \& Gullbring 1998). In the following we will call outflow both disk-wind and jet. The inner outflow region, close to the disk ( $<1$ AU from the disk), will be called disk-wind and the outer collimated outflow region will be referred to as a jet.

It has always been clear that both wind and magnetospheric accretion are important processes acting in the young star-disk systems and contributing to line formation (Bertout 1989; Appenzeller et al. 2005), both in absorption and emission. Muzerolle et al. (2001) also suggested that some CTTSs with high mass accretion rates, like DR Tau, may power powerful outflows and most of the $\mathrm{H} \alpha$ line may indeed be produced in winds due to high optical depth. In this case, the permitted emission lines may be used as new constraints to the wind characteristics. Therefore the study of the wind in young stellar objects is necessary to complement the magnetospheric accretion scenario.

RW Aur (HBC 80; K1) is a bright $\left(V_{\max }=10.1 \mathrm{mag}\right)$ CTTS that presents high values of veiling $(0.3<v<$ 6.1, Stout-Batalha et al. 2000) and mass accretion rate $\left(\log \left(\dot{M}_{\mathrm{acc}} / M_{\odot} \mathrm{yr}^{-1}\right) \sim-7.5\right.$ to -6 , White \& Ghez 2001; Valenti et al. 1993; Hartigan et al. 1995), together with strong, variable emission lines. RW Aur is a member of the Taurus-Auriga star forming region and it is often quoted as a triple system with the primary component A separated by $1.4^{\prime \prime}$ from components $\mathrm{B}$ and $\mathrm{C}$. However, component $\mathrm{C}$ was not detected by White \& Ghez (2001) in their HST images, although the expected flux of component $\mathrm{C}$ were much above their detection limit, and therefore they considered component $\mathrm{C}$ a false detection from earlier data (Ghez et al. 1993).

Component A presents periodic variability in its spectral and broad emission lines and is suspected to be a single-lined spectroscopic binary with a 2.77-day period (Gahm et al. 1999; Petrov et al. 2001). This component also powers a variable outflow associated with a bipolar micro-jet, inferred from the presence of both blue and red high velocity components in the forbidden [OI](6300 ̊) line (Hartigan et al. 1995; Hirth et al. 1997), and later imaged with adaptive optics (Dougados et al. 2000; López-Martín et al. 2003) and STIS-HST (Woitas et al. 2002) down to about 10 AU from the star. Recently it was shown that the RW Aur jet close to the star rotates
(Coffey et al. 2004; Woitas et al. 2005), and the measured toroidal velocities are similar to the values predicted by magneto-centrifugal models of winds, giving strong support to this class of models.

In this paper we present the analysis of the main optical emission lines of RW Aur. Our goal is to investigate the importance of winds and the dynamics of the accretion and outflow processes in this system.

\section{Observations}

We present the analysis of a sample of 77 spectra of the CTTS RW Aur listed in Table 1. The observations, obtained over a decade, were carried out at Lick Observatory, some using the $3 \mathrm{~m}$ Shane reflector, most using the $0.6 \mathrm{~m}$ Coudé Auxiliary Telescope (CAT) to feed the Hamilton Echelle Spectrograph (Vogt 1987) coupled either to a TI $800 \times 800 \mathrm{CCD}$ or a FORD $2048 \times 2048$ CCD. The entire spectral format is not covered with the smaller CCD, so observations were generally obtained in one of two settings: 1 ) a red setting covering 52 partial orders from $\sim 4900 \AA$ to $\sim 8900 \AA$; and 2) a blue setting covering 38 partial orders from $\sim 3900 \AA$ to $\sim 5200 \AA$. Whenever possible, blue and red observations were obtained in the same night or on successive nights. The larger CCD, installed in 1992, records $\sim 92$ orders covering the optical spectrum from $\sim 3900 \AA$ to $\sim 8900 \AA$. The mean resolution of the spectra is $\lambda / \Delta \lambda \approx 48000$.

The reduction was performed in a standard way described by Valenti (1994) which includes flatfielding with an incandescent lamp exposure, background subtraction, and cosmic ray removal. Wavelength calibration is made by observing a Thorium-Argon comparison lamp and performing a 2D solution to the position of the Thorium lines as a function of order and column number. Radial and barycentric velocity corrections have been applied, and all the data shown here are in the stellar rest frame. No flux calibration has been attempted for these spectra; rather each spectrum has been continuum normalized. Due to differences in weather conditions, exposure times, telescope used, and efficiency between the different $\mathrm{CCDs}$, there is a wide range in the signal-to-noise ratio $(\mathrm{S} / \mathrm{N})$ in the data. Part of the data presented here were previously published and analyzed in another context (Basri \& Batalha 1990; Johns \& Basri 1995a; Stout-Batalha et al. 2000).

\section{Analysis}

\subsection{Profile variability}

We show in Fig. 1 the mean line profiles of $\mathrm{H} \alpha, \mathrm{H} \beta$, He I (5876 ̊), NaD and Ca II (8498 $\mathrm{A})$ which were obtained by taking the mean value in each velocity bin of a given line over all the observations. Also shown in the shaded area are the normalized variance profiles which measure the amount of variability in each velocity bin in the line profile. As defined by Johns \& Basri (1995a), the temporal variance of the line at each velocity bin is given by

$\sum_{v}=\left[\frac{\sum_{i=1}^{n}\left(I_{v, i}-\bar{I}_{v}\right)^{2}}{(n-1)}\right]^{1 / 2}$ 
Table 1. Journal of observations. JD is the Julian Date of the observation.

\begin{tabular}{|c|c|c|c|c|c|}
\hline Obs. & UT date and time & JD-2 400000.0 & Obs. & UT date and time & JD-2 400000.0 \\
\hline $1 \ldots .$. & 1989 Jan. 04, 05:13 & 47530.717 & $40 \ldots$ & 1992 Oct. $25,12: 10$ & 48921.007 \\
\hline $2 \ldots \ldots$ & 1989 Jan. 04, 08:37 & 47530.860 & $41 \ldots$ & 1992 Oct. $26,11: 24$ & 48921.975 \\
\hline $3 \ldots .$. & 1989 Jan. 04, 10:46 & 47530.949 & $42 \ldots$ & 1992 Oct. $29,08: 35$ & 48924.858 \\
\hline $4 \ldots .$. & 1989 Jan. $18,06: 58$ & 47544.791 & $43 \ldots$ & 1992 Nov. 03, 10:37 & 48929.943 \\
\hline $5 \ldots .$. & 1989 Jan. 21, 08:25 & 47547.851 & $44 \ldots$ & 1992 Nov. $15,11: 54$ & 48941.996 \\
\hline $6 \ldots .$. & 1989 Jan. 22, 02:49 & 47548.618 & $45 \ldots$ & 1992 Nov. 16, 09:31 & 48942.897 \\
\hline $7 \ldots \ldots$ & 1989 Jan. 22, 06:10 & 47548.757 & $46 \ldots$ & 1992 Nov. 17, 11:49 & 48943.993 \\
\hline $8 \ldots .$. & 1989 Jan. 22, 09:04 & 47548.878 & $47 \ldots$ & 1992 Nov. 24, 09:21 & 48950.890 \\
\hline $9 \ldots .$. & 1989 Jan. 23, 02:40 & 47549.611 & $48 \ldots$ & 1992 Nov. 25, 11:51 & 48951.994 \\
\hline $10 \ldots$ & 1989 Jan. 23, 05:47 & 47549.741 & $49 \ldots$ & 1992 Nov. 26, 08:52 & 48952.869 \\
\hline $11 \ldots$. & 1989 Jan. 23, 09:10 & 47549.882 & $50 \ldots$ & 1992 Nov. 26, 10:10 & 48952.924 \\
\hline $12 \ldots$. & 1989 Nov. $12,08: 43$ & 47842.863 & $51 \ldots$ & 1992 Nov. 27, 08:32 & 48953.856 \\
\hline $13 \ldots$ & 1989 Nov. $12,13: 01$ & 47843.043 & 52. & 1992 Nov. $28,11: 53$ & 48954.995 \\
\hline $14 \ldots$. & 1990 Oct. $22,10: 23$ & 48186.933 & $53 \ldots$ & 1992 Nov. 29, 08:26 & 48955.851 \\
\hline $15 \ldots$ & 1990 Oct. $22,12: 47$ & 48187.033 & $54 \ldots$ & 1992 Nov. 30, 07:37 & 48956.818 \\
\hline $16 \ldots$ & 1990 Oct. $23,08: 40$ & 48187.861 & $55 \ldots$ & 1992 Dec. 01, 08:32 & 48957.856 \\
\hline $17 \ldots$. & 1990 Oct. $24,11: 44$ & 48188.989 & $56 \ldots$ & 1992 Dec. 02, 08:40 & 48958.861 \\
\hline $18 \ldots$ & 1990 Oct. $25,09: 53$ & 48189.912 & $57 \ldots$ & 1992 Dec. 03, 09:00 & 48959.875 \\
\hline $19 \ldots$. & 1990 Oct. $26,08: 37$ & 48190.859 & $58 \ldots$ & 1992 Dec. 20, 07:52 & 48976.828 \\
\hline $20 \ldots$ & 1990 Oct. $28,10: 06$ & 48192.921 & $59 \ldots$ & 1992 Dec. 22, 06:51 & 48978.785 \\
\hline $21 \ldots$. & 1990 Nov. $14,11: 26$ & 48209.977 & $60 \ldots$ & 1992 Dec. 23, 07:45 & 48979.824 \\
\hline $22 \ldots$. & 1990 Nov. 15, 09:01 & 48210.876 & $61 \ldots$ & 1992 Dec. $24,07: 42$ & 48980.822 \\
\hline $23 \ldots$ & 1990 Nov. $17,07: 32$ & 48212.815 & $62 \ldots$ & 1992 Dec. 25, 07:31 & 48981.814 \\
\hline $24 \ldots$. & 1990 Nov. 19, 07:03 & 48214.794 & $63 \ldots$ & 1993 Feb. 06, 07:41 & 49024.821 \\
\hline $25 \ldots$. & 1991 Oct. $25,08: 49$ & 48554.868 & $64 \ldots$ & 1993 Feb. 14, 07:36 & 49032.817 \\
\hline $26 \ldots$ & 1991 Oct. $25,11: 23$ & 48554.974 & $65 \ldots$ & 1999 Nov. 22, 09:05 & 51504.879 \\
\hline $27 \ldots$. & 1991 Oct. $25,13: 07$ & 48555.047 & $66 \ldots$ & 1999 Nov. 23, 06:56 & 51505.789 \\
\hline $28 \ldots$. & 1992 Jan. 09, 10:19 & 48630.931 & $67 \ldots$ & 1999 Nov. 24, 08:45 & 51506.865 \\
\hline $29 \ldots$ & 1992 Jan. 11, 07:24 & 48632.809 & $68 \ldots$ & 1999 Nov. 25, 08:04 & 51507.837 \\
\hline $30 \ldots$ & 1992 Jan. 13, 08:27 & 48634.853 & $69 \ldots$ & 1999 Nov. 26, 07:47 & 51508.824 \\
\hline $31 \ldots$. & 1992 Jan. 14, 08:34 & 48635.857 & $70 \ldots$ & 1999 Nov. 27, 08:49 & 51509.867 \\
\hline $32 \ldots$. & 1992 Jan. 15, 06:34 & 48636.774 & $71 \ldots$ & 1999 Nov. 28, 08:42 & 51510.863 \\
\hline $33 \ldots$. & 1992 Jan. 16, 07:36 & 48637.817 & $72 \ldots$ & 1999 Dec. 11, 08:09 & 51523.840 \\
\hline $34 \ldots$. & 1992 Jan. 17, 08:15 & 48638.844 & $73 \ldots$ & 1999 Dec. $12,09: 35$ & 51524.900 \\
\hline $35 \ldots$. & 1992 Sep. 22, 12:29 & 48888.020 & $74 \ldots$ & 1999 Dec. 15, 07:57 & 51527.832 \\
\hline $36 \ldots$ & 1992 Sep. $26,12: 13$ & 48892.010 & $75 \ldots$ & 1999 Dec. 16, 07:50 & 51528.827 \\
\hline $37 \ldots$. & 1992 Oct. $21,12: 44$ & 48917.031 & $76 \ldots$ & 1999 Dec. 17, 07:30 & 51529.813 \\
\hline $38 \ldots$. & 1992 Oct. $23,12: 32$ & 48919.022 & $77 \ldots$ & 1999 Dec. 18, 07:27 & 51530.811 \\
\hline $39 \ldots$. & 1992 Oct. $24,11: 48$ & 48919.992 & & & \\
\hline
\end{tabular}

where $n$ is the number of observations, $I_{v, i}$ is the profile intensity at a given velocity $(v)$ in each observation $(i)$ and $\bar{I}_{v}$ is the mean intensity at a given velocity $(v)$ over all the observed profiles. The normalized variance profile is the variance profile as defined above divided by the average line profile.

In Fig. 2 are overplotted all the observations of the above emission lines that will be analyzed. Unfortunately most of the $\mathrm{H} \beta$ profiles fall in the edge of the CCD and are cut in the early spectra, the He I ( $5876 \AA$ ) line often presents low S/N and many $\mathrm{NaD}$ lines show a strong contamination from the city light of San Jose. Due to that, except for $\mathrm{H} \alpha$, we will only analyze the Nov. 99 + Dec. 99 observing runs.

$\mathrm{H} \alpha$ presents a very wide mean emission line profile that goes from $-500 \mathrm{~km} \mathrm{~s}^{-1}$ to $+500 \mathrm{~km} \mathrm{~s}^{-1}$ with a strong absorption centered around $-100 \mathrm{~km} \mathrm{~s}^{-1}$. The amplitude of the variations is about the same in the long period of observations from Jan. 89 to Feb. 93 and in the two periods of about one week in Nov. $99+$ Dec. 99. Strong intensity variations can occur in one day, but the maximum amplitude of variability is overall constant. The far red wing varies less than the rest of the profile and we can easily see that the blueshifted absorption in $\mathrm{H} \alpha$ varies (in amplitude) much less than the other parts of the profile too, like in SU Aur (Johns \& Basri 1995b). We have to keep in mind that the normalized variance in absorption features looks higher because it is divided by a smaller number. That is why the blueshifted absorption varies less in amplitude than the emission peaks but still presents a significant normalized variance profile. It is actually rather common that some regions of the emission profiles of CTTSs stay almost invariant because the integrated line emission seen at their velocities remains constant over the rotation period, as pointed out by Symington et al. (2005). This could be due to the fact that these velocities are dominated, for example, by material in outer regions of the wind, like the blueshifted absorption that may 

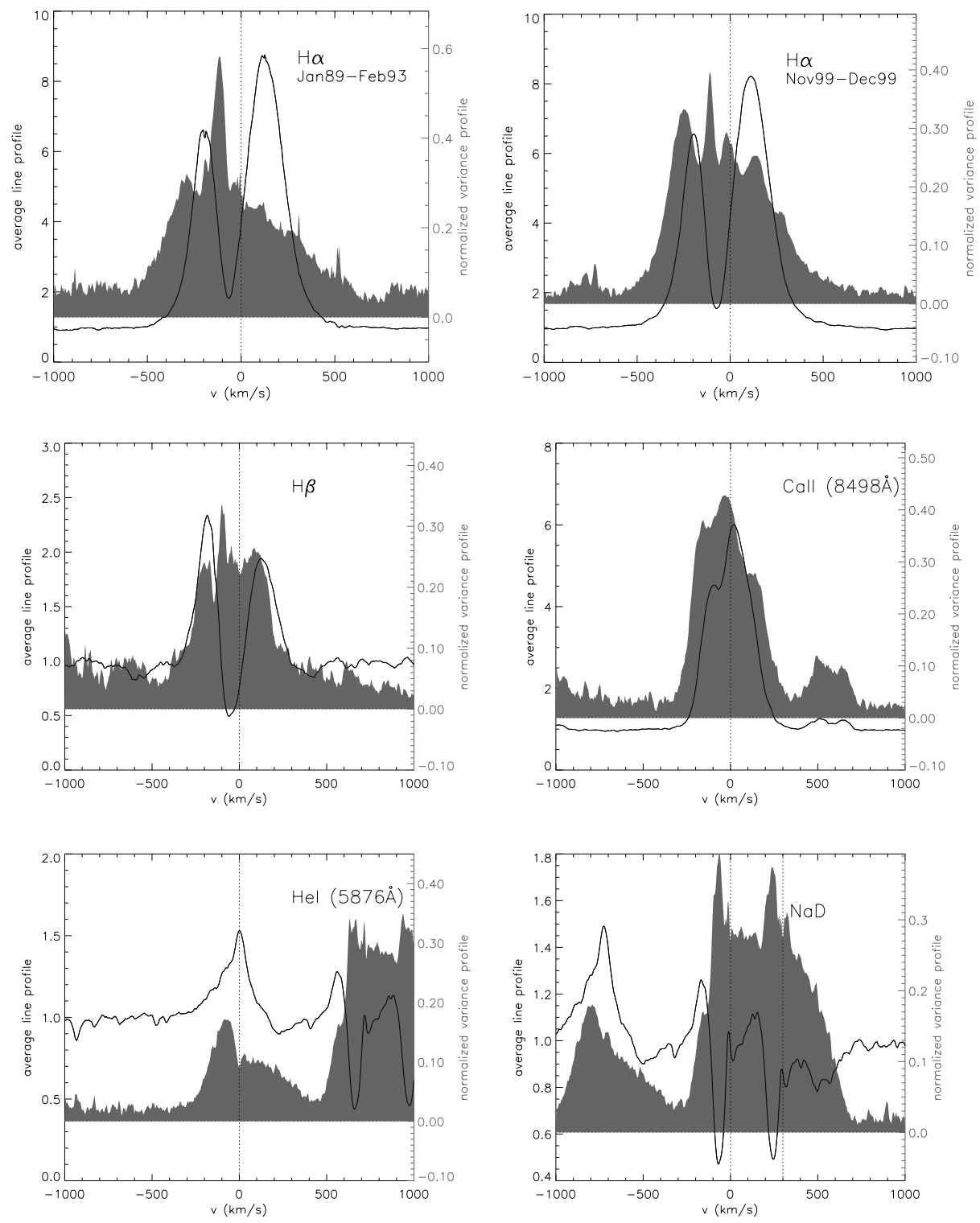

Fig. 1. Mean (solid black line) and variance (grey shaded area) profiles. Except for $\mathrm{H} \alpha$, the mean and variance profiles were calculated only with the Nov. $99+$ Dec. 99 data.

originate in the outer cold wind, or the far red emission of $\mathrm{H} \alpha$ that could be partly formed in the outer receding jet lobe, as discussed in the following sections.

We consider the lack of flux around $-100 \mathrm{~km} \mathrm{~s}^{-1}$ in $\mathrm{H} \alpha$ to be partly caused by a true absorption due to winds instead of being just a lack of emission, which would correspond to a case where the two emission peaks would belong to completely separate emission events. Our interpretation is based on the fact that $\mathrm{H} \beta$ mimics $\mathrm{H} \alpha$, with the huge blueshifted absorption now clearly going below the continuum. Three $\mathrm{H} \alpha$ spectra actually show the blueshifted absorption going below the continuum too ( $\mathrm{JD}=48554.868,48554.974,48555.047)$. These correspond to spectra presenting specially intense $\mathrm{H} \beta$ blue absorptions and it really points out to a wind origin for the blueshifted absorption in both $\mathrm{H} \beta$ and $\mathrm{H} \alpha$.

He I (5876 ̊) shows broad (BC) and narrow (NC) emission components and redshifted absorption components. The $\mathrm{BC}$ sometimes increases significantly in the blue side and varies more than the rest of the He I (5876 $\AA$ ) line profile, similar to the results of Beristain et al. (2001). According to them, in high mass accretion rate CTTSs that present highly blueshifted $\mathrm{BCs}$, the blue emission of the $\mathrm{BC}$ could be mostly due to winds, that are also supposed to influence the $\mathrm{H} \alpha$ blue emission. The three spectra that present the most intense BC blue emission ( $\mathrm{JD}=48$ 189.912, 51 509.867, 51 530.811) also show strong $\mathrm{H} \alpha, \mathrm{H} \beta, \mathrm{Ca}$ II $(8498 \AA)$ and [OI](6300 $⿱$ ) emission. But there is no clear evidence that only the blue side of $\mathrm{H} \alpha$ is enhanced. It is rather as if the entire line is more intense. So the strong He I (5876 A) BC blue emission could be clearly associated to winds in RW Aur if most of the main emission lines have their origin in the wind too and not only part of the blue $\mathrm{H} \alpha$ emission, as suggested by Beristain et al. (2001).

The main absorption in the $\mathrm{NaD}$ lines is blueshifted and we clearly see that a redshifted absorption comes and goes 

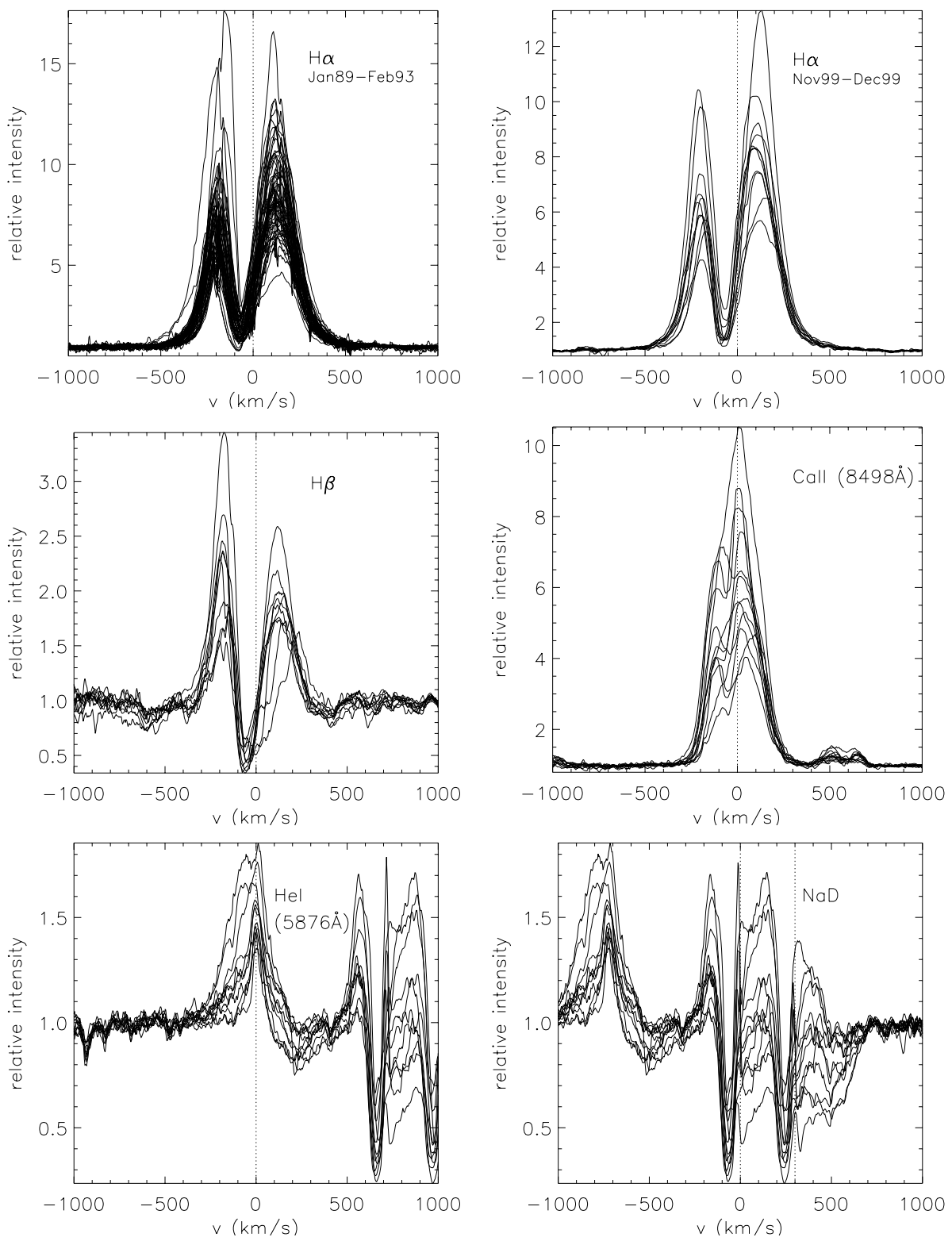

Fig. 2. Line profiles overplotted. Except for H $\alpha$, only the Nov. 99 + Dec. 99 profiles are plotted.

away. The presence of redshifted absorption components at 200-300 km s${ }^{-1}$ in some of the lines reinforces the idea that accretion is present in RW Aur and that the system inclination cannot be too close to pole-on.

Ca II (8498 ̊) shows a very intense emission with a blueshifted absorption centered at approximately the same velocity as the $\mathrm{H} \alpha$ blue absorption. Like $\mathrm{H} \alpha$ again, the far red wing varies less than the rest of the profile. The blueshifted absorption in the Ca II (8498 $⿱$ ) profiles is normally present, but some spectra show a much shallower absorption than others.

In Fig. 3 we show three chosen Ca II (8662 Å) line profiles that present particularly different morphologies. We would like to point out that one has to be careful not to ascribe the profile features just to system geometry or general morphology - clearly the same system can exhibit rather different profiles at different times. The physical interpretation given to each of these profile types is usually rather distinct. This shows the need for time variable accretion and outflow models of CTTSs.

The veiling in RW Aur is high and variable (e.g. $0.3<$ veiling < 6.1, Stout-Batalha et al. 2000). However, if most of the observed line variabilities were due to veiling, the variance profiles would reproduce the mean line profile shape and this is not the case. We did not attempt to measure veiling in the CAT spectra, since the $\mathrm{S} / \mathrm{N}$ is not high enough for a confident measurement. Most of the $3 \mathrm{~m}$ spectra were already previously analyzed (Stout-Batalha et al. 2000) and had their veiling values carefully determined.

\subsection{Periodogram analysis}

We searched for periodical changes in the emission line intensities. Since our data are scattered in many different observing runs, the best way to do the period search is to look at individual 


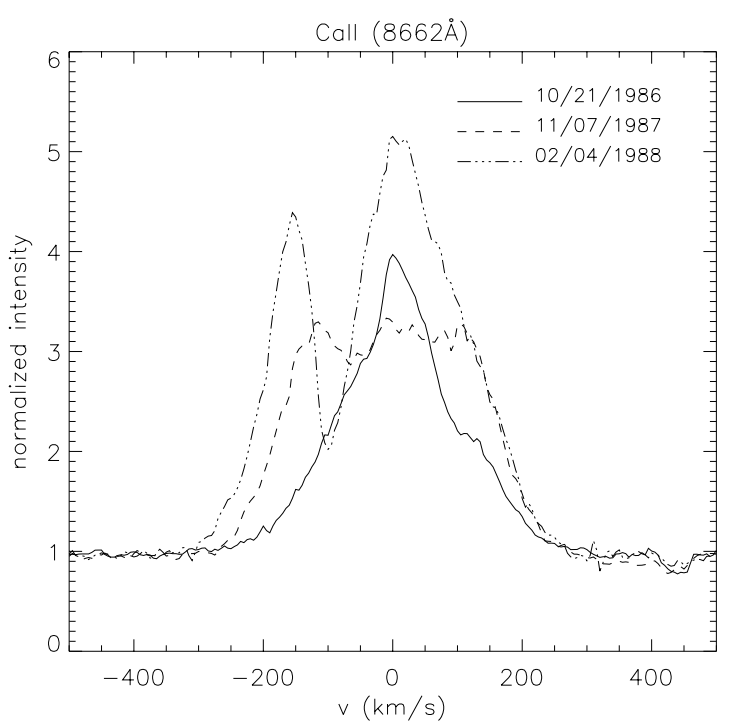

Fig. 3. Ca II ( $8662 \AA)$ lines observed at different epochs.

runs separately. We looked for periods whenever we had observations covering at least 6 days. The results obtained are displayed in Fig. 4.

Petrov et al. (2001) analyzed three series of high-resolution spectra of RW Aur from 1996, 1998 and 1999. They found periodic variabilities at about 2.77 days in the weak absorption lines and in narrow emission lines. The broad emission lines in their spectra show periodic changes at about 5.5 days and also present the 2.77-day variabilities but with less power. They propose two possible models to explain their data, one where RW Aur is a binary with a brown dwarf companion with an orbital period of 2.77 days and another which assumes that RW Aur is a single star with a rotational period of 5.5 days and two major hotspots due to accretion from a non-axisymmetric dipole configuration. In the latter the hotspots would be responsible for the detected 2.77 day period.

Our datasets of Nov. 92 and Nov. 99+Dec. 99 allow us to investigate both the 2.77 and the 5.5-day periods. Unexpectedly, in the former we find the 2.77 and a 3.9-day period and in the latter a 4.2 and the 5.5-day period. The 3.9-day period has even a higher detection power than the 2.77-day one in the Nov. 92 periodogram. The 4.2 and 5.5-day periods found in the Nov. $99+$ Dec. 99 dataset are present in all the main emission lines $(\mathrm{H} \alpha, \mathrm{H} \beta, \mathrm{Ca}$ II $(8498 \AA)$, He I (5876 $\AA)$ ) and the 5.5-day period is more significant than the 4.2-day one, presenting a higher power in the periodogram. Our Nov. $99+$ Dec. 99 results agree fairly well with those by Petrov et al. (2001) but the broad emission lines observed in Nov. 92 do not present the expected 5.5 day periodicity, although they show the 2.77 day period but with very low power. If the observed periods are due to hot spots at the stellar surface, the lack of the 5.5 day periodicity in the earlier data could indicate a change in the magnetospheric configuration of RW Aur from the early to the late 90 's, corresponding to a different spot configuration between the two epochs.
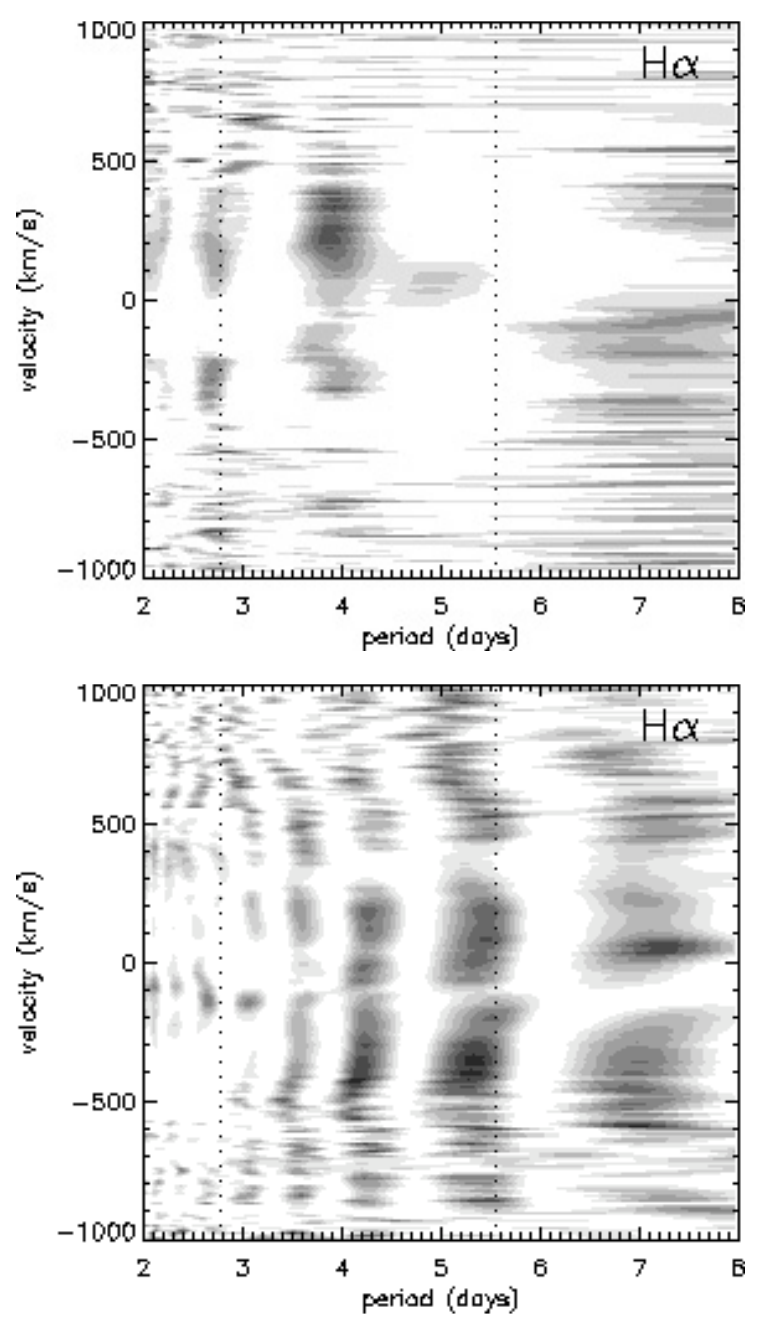

Fig. 4. $\mathrm{H} \alpha$ periodograms. Top: Nov. 92 dataset. Bottom: Nov. $99+$ Dec. 99 dataset. The dotted lines correspond to the periods detected by Petrov et al. (2001), 2.77 and 5.5 days.

\subsection{Correlation matrices}

In order to investigate how the profile variations are correlated across a given emission line, we calculated autocorrelation matrices (Johns \& Basri 1995a) for the main emission lines using the dataset of Nov. $99+$ Dec. 99. A sample of the resulting matrices are displayed in Fig. 5 and are discussed below.

The $\mathrm{H} \alpha$ result shows that the inner blue wing $\left(-150 \mathrm{~km} \mathrm{~s}^{-1}<v<20 \mathrm{~km} \mathrm{~s}^{-1}\right)$ and the far red wing $\left(v>200 \mathrm{~km} \mathrm{~s}^{-1}\right)$ do not correlate with the blue and red emission peaks. It points to a different origin of these $\mathrm{H} \alpha$ regions with respect to the rest of the profile. This was also suggested in Sect. 3.1, since these line regions vary much less than the rest of the profile. In Sect. 3.5 we show that we can reproduce most of the Balmer line emission with a disk-wind, except for the outer red wing, that may have an important contribution from the receding jet lobe or be partly due to damping, and part of the blueshifted absorption, that is thought to come from the outer cold wind, which we did not model, rather than close to the star.

The He I (5876 A) blue emission correlates well with itself but does not correlate with the red side of the profile, indicating 

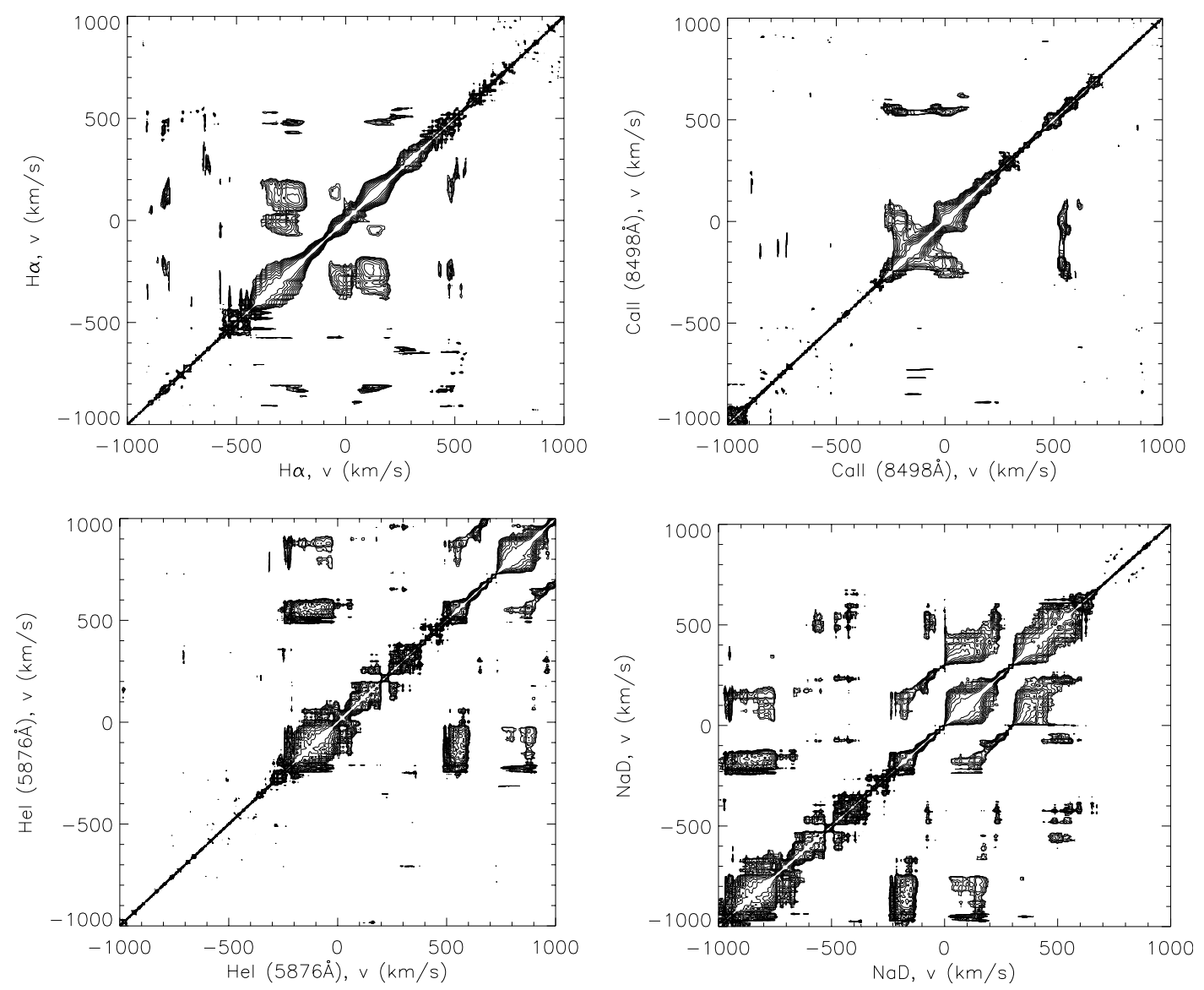

Fig. 5. H $\alpha$, Ca II ( $8498 \AA)$, He I ( $5876 \AA$ ) and NaD autocorrelation matrices calculated with the Nov. $99+$ Dec. 99 data.

that the He I (5876 ̊) blue and red sides are mostly influenced by different processes. The blue emission is thought to be mainly due to winds and the accretion process is thought to be responsible for the redshifted absorptions in the red wing as proposed by Beristain et al. (2001).

The NaD doublet is difficult to analyze in RW Aur, since both lines present a blue and a red emission, a blue absorption and often a redshifted absorption too. Due to the proximity of the lines, the redshifted emission or absorption of the D1 line is mixed up with the blueshifted emission of the D2 line. What can be said about the correlation matrix is that the $0 \mathrm{~km} \mathrm{~s}^{-1}<v<200 \mathrm{~km} \mathrm{~s}^{-1}$ region correlates well with the $300 \mathrm{~km} \mathrm{~s}^{-1}<v<500 \mathrm{~km} \mathrm{~s}^{-1}$ region. These correspond to the regions mostly affected by the accretion process, since these are the regions where the redshifted absorptions appear. The almost total lack of correlation across the rest of the line indicates that the blue emission and absorption components of these lines are probably not related to accretion but rather to outflow. This is confirmed by the good correlation between the blue emission of the D1 line and the He I (5876 ̊) BC blue emission that is thought to be due to winds and corresponds to the region $-1000 \mathrm{~km} \mathrm{~s}^{-1}<v<-700 \mathrm{~km} \mathrm{~s}^{-1}$ in the $\mathrm{NaD}$ autocorrelation matrix (see Fig. 2, lower right panel to locate the He I (5876 ̊) line in the $\mathrm{NaD}$ velocity frame).

Like the $\mathrm{H} \alpha$ line, the $\mathrm{Ca}$ II (8498 $\AA$ ) line also shows an overall good correlation across the line, except for the blueshifted absorption and the far red wing regions, indicating that these two regions come probably from different formation sites than most of the emission profile.

We then calculated correlation matrices between $\mathrm{H} \alpha$ and the other emission lines in order to try to identify which line components have a common origin. The resulting correlation matrices are displayed in Fig. 6 and discussed below.

$\mathrm{H} \alpha$ and $\mathrm{H} \beta$ present correlations between their outer blue emission peaks and their inner red emission peaks. The inner blue regions of the two lines do not correlate. This is the region corresponding to the blue absorption and it is also affected by the emission due to the accretion process, as will be discussed in Sect. 3.5. The outer red emission wing of $\mathrm{H} \alpha$ does not correlate either with $\mathrm{H} \beta$.

$\mathrm{H} \alpha$ and Ca II (8498 $\AA$ ) are well correlated with each other, except again for the outer red wings of both lines and the absorption region. The He I ( $5876 \AA$ ) blue emission is well correlated with the $\mathrm{H} \alpha$ blue and red emissions, except for the $\mathrm{H} \alpha$ outer red wing $\left(v>200 \mathrm{~km} \mathrm{~s}^{-1}\right)$.

The blue emission of the D1 line correlates well with $\mathrm{H} \alpha$, except again for the outer red wing of $\mathrm{H} \alpha$, indicating a common origin for both emission components. The D2 line emission is very much affected by the superposed redshifted absorption of the D1 line and therefore does not show much of a correlation with $\mathrm{H} \alpha$, as expected.

The correlations found in this section will be discussed in more detail in Sects. 3.5 and 4. 

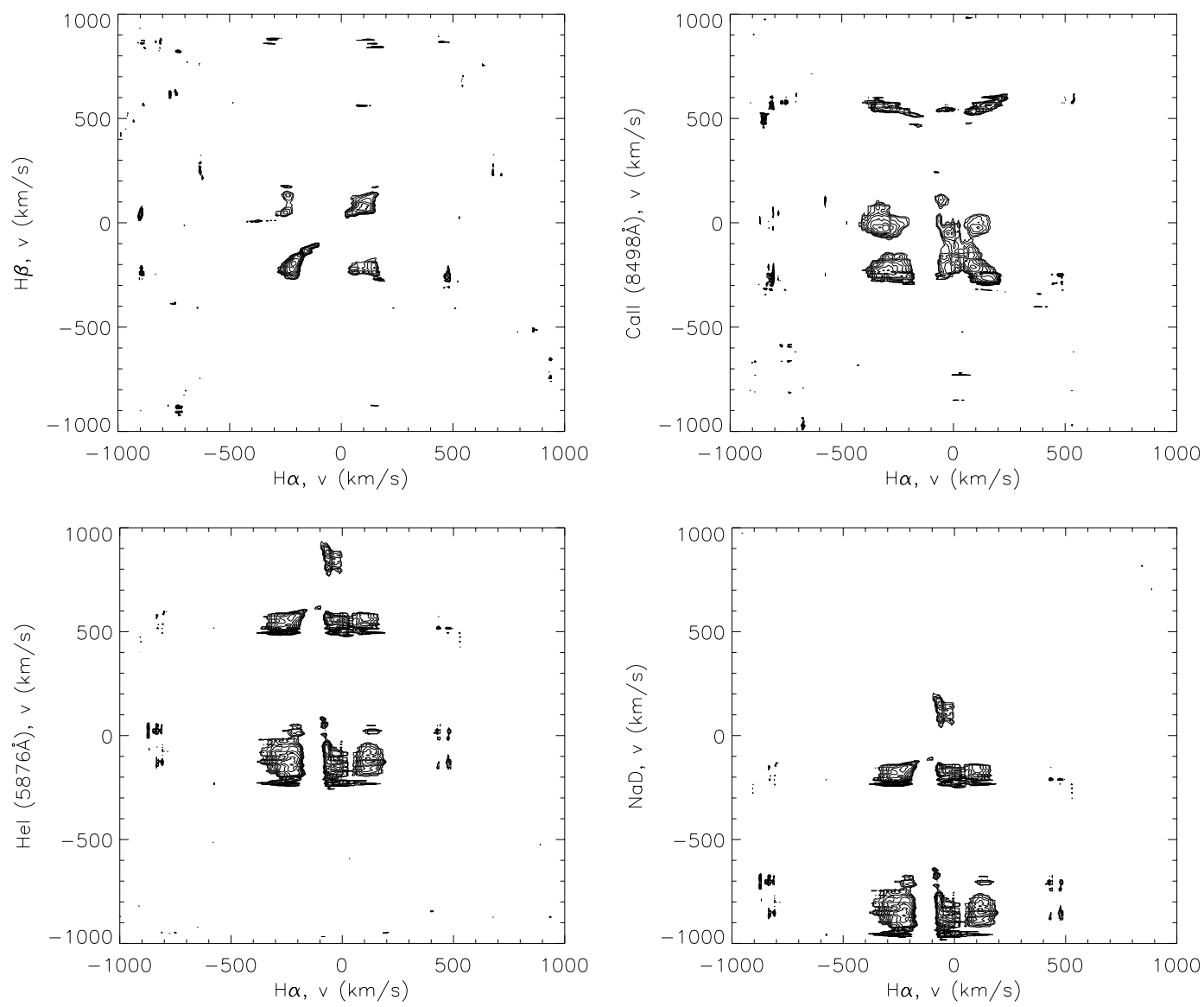

Fig. 6. Correlation matrices between $\mathrm{H} \alpha$ and the main emission lines calculated with the Nov. $99+$ Dec. 99 data.

\subsection{Forbidden emission lines}

One way to investigate the wind/outflow characteristics of RW Aur is to analyze the [OI] $(6300 \AA)$ forbidden emission line. In high mass accretion rate CTTSs this line normally presents two distinct components, a high velocity one (HVC) attributed to the jet and a low velocity component (LVC) that is thought to be due to the disk-wind (Hartigan et al. 1995). Unfortunately in many of our early spectra the $[\mathrm{OI}](6300 \AA)$ line is between échelle orders and in some there are bad pixels that fall right on top of it. The Nov. $99+$ Dec. 99 data is the only run that does not present any of the problems above and the corresponding [OI](6300 A) are displayed in Fig. 7. The RW Aur [OI](6300 A) line looks very much like the one observed by Hartigan et al. (1995), presenting three emission peaks with two high velocity components, the blue HVC generally less intense and wider than the red one.

We decomposed the $[\mathrm{OI}](6300 \AA)$ line using Gaussian emissions and measured the central velocity and equivalent widths of each component. As can be seen in Fig. 8, the LVC is slightly blueshifted by $\sim-10 \mathrm{~km} \mathrm{~s}^{-1}$, the HVC blue peak is centered at about $-170 \mathrm{~km} \mathrm{~s}^{-1}$ and the red peak around $+120 \mathrm{~km} \mathrm{~s}^{-1}$. All the components show a daily variability in their peak velocities, the red HVC presenting a much smaller velocity variation than the blue one. The blue/red velocity asymmetry noticed by several authors between the two jet lobes holds within the variability, since $\left|v_{\mathrm{HVCblue}}\right| /\left|v_{\mathrm{HVCred}}\right|>1$ in all the observations.
The $[\mathrm{OI}](6300 \AA)$ high velocity components are thought to come from the jet. Dougados et al. (2000) imaged the RW Aur A jet in [OI] $(6300 \AA)$ and [SII] $(6735 \AA)$ using adaptive optics at CFHT in December 1997 and saw both a redshifted and a blueshifted jet. They detected the redshifted jet down to $0.4^{\prime \prime}$ (56 AU) from the star. López-Martín et al. (2003) showed that in December 1998, with a resolution of $0.4^{\prime \prime}$, both the blue and redshifted jets presented knots closer than $1^{\prime \prime}$ from the star. This was also confirmed by Woitas et al. (2002) using HST/STIS data with observations from December 2000. Consequently, at least in our Nov. 99 + Dec. 99 observations, we certainly got the contribution of both jet sides, since they were seen closer than $2^{\prime \prime}$ (our typical seeing at Lick) from the star, one year before and one year after we observed RW Aur. This is why we see two high velocity peaks in the [OI](6300 $\AA$ ) line of RW Aur, each corresponding to a given jet lobe.

The observations mabe by Woitas et al. (2002), from December 2000, do not present the low velocity component (LVC) of the $[\mathrm{OI}](6300 \AA)$ line that is clearly seen in our spectra of Nov. $99+$ Dec. 99 and is actually more intense than the blue HVC (see Fig. 7). They say that the lack of LVC in their data and the presence of it in the profiles obtained by Hartigan et al. (1995) and Hirth et al. (1997) in 1990 and 1993 respectively, indicate temporal variability on a timescale of a few years. However, the timescale of the variability is actually much shorter, since the LVC is clearly present in our data obtained just one year before theirs. If the LVC is really associated 


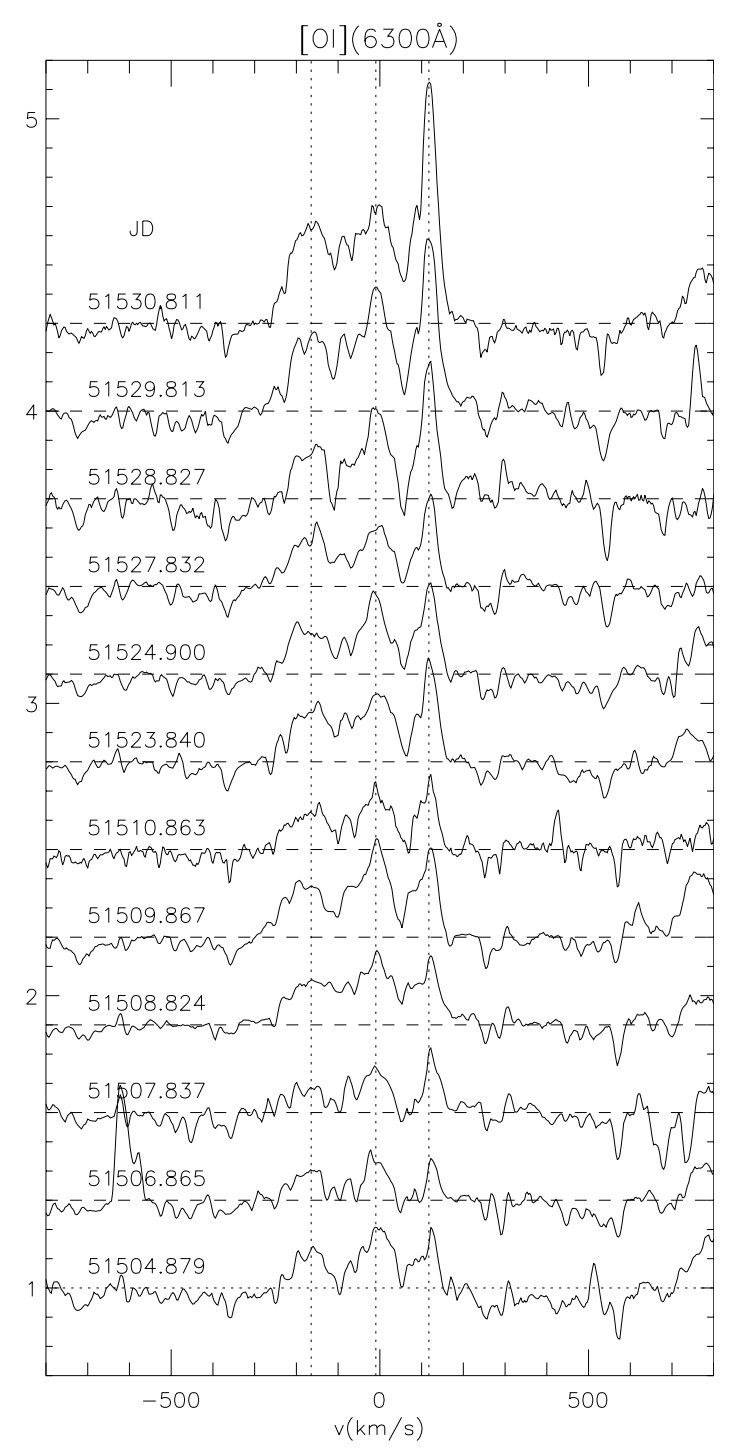

Fig. 7. $[\mathrm{OI}](6300 \AA)$ lines observed in the Nov. $99+$ Dec. 99 run. The dashed lines show the normalized continuum and the dotted lines represent the mean radial velocity of the three line components (LVC and blue and red HVC).

with the disk-wind, as suggested by the models of Kwan \& Tademaru $(1988,1995)$, then the RW Aur A disk-wind can vary dramatically in a short period of a year or less, perhaps due to magnetic instabilities as proposed by Goodson et al. (1999) and Romanova et al. (2003). As can be seen in Figs. 7 and 9, the intensities and equivalent widths of the three emission components of $[\mathrm{OI}](6300 \AA)$ also smoothly vary on a daily basis, showing the outflow is a dynamical process even on a timescale of hours. The presence of daily variations implies that the region responsible for the variable emission in the jet cannot be greater in extent than a few times ten stellar radii, which corresponds to a few times the distance covered at the jet velocity in a day. In the first two panels of Fig. 9 we show equivalent width ratios between the LVC and the HVC. The fact that the variations of the three components are independent one from the other shows that the observed line variability cannot be due to veiling alone.
The equivalent width ratio of the LVC to the HVC in our data is in the range $0.4<E W(\mathrm{LVC}) / E W(\mathrm{HVC})<0.8$, as can be seen in Fig. 9 (right panel). Hartigan et al. (1995) measured in four spectra the total equivalent width of the $[\mathrm{OI}](6300 \AA)$ line and the equivalent width of the $|v| \leq$ $60 \mathrm{~km} \mathrm{~s}^{-1}$ region, that can be associated to the LVC and found $0.3<E W(\mathrm{LVC}) / E W(\mathrm{HVC})<0.5$. Hirth et al. (1997) obtained $E W(\mathrm{LVC}) / E W(\mathrm{HVC})=0.14$, but we redid their profile decomposition and obtained instead $E W(\mathrm{LVC}) / E W(\mathrm{HVC})=0.39$. We do not know the origin of such a discrepancy of values since we both used a Gaussian decomposition to determine the contribution of each component. These equivalent width ratios show that the disk-wind flux in RW Aur is comparable to the flux carried by the jet. Since the disk-wind is supposed to have much higher densities than the jet, as can be inferred by the presence of the LVC in [OI] lines but not in [SII] lines, we would therefore expect that the disk-wind may significantly contribute to most of the permitted emission lines as well.

\subsection{Accretion and wind models}

RW Aur is a high mass accretion rate system $\left(\log \left(\dot{M}_{\mathrm{acc}} / M_{\odot} \mathrm{yr}^{-1}\right) \sim-7.5\right.$ to -6 , White \& Ghez 2001; Valenti et al. 1993; Hartigan et al. 1995) that has Balmer emission lines which overall do not look like magnetospheric accretion line profiles. The RW Aur line profiles can be compared with magnetospheric accretion profiles calculated by Muzerolle et al. (2001) for a large variety of parameters and canonical $\mathrm{T}$ Tauri values of mass and radius $\left(0.5 \mathrm{M}_{\odot}\right.$, $2 R_{\odot}$ ). Following Hartmann et al. (1994), we calculated magnetospheric accretion profiles for RW Aur, using as stellar parameters $M=1 M_{\odot}, R=2 R_{\odot}$ and $T_{\text {eff }}=4600 \mathrm{~K}$ (Stout-Batalha et al. 2000). We computed profiles with two different temperature levels, characterized by the maximum value of the temperature distribution, $T_{\max }=6000 \mathrm{~K}$ and $7000 \mathrm{~K}$, following the suggestion by Muzerolle et al. (2001) that these values generate profiles which present overall the characteristics of the observed CTTS line profiles for a mass accretion rate of the order of $10^{-6} M_{\odot} \mathrm{yr}^{-1}$. We set the accretion ring temperature at $T_{\text {shock }}=8000 \mathrm{~K}$. The inner and outer magnetosphere radii are free parameters but we know that accretion can only occur inside the co-rotation radius (Shu et al. 1994). With the above stellar parameters and supposing the stellar magnetic field to be of the order of 1 to $2 \mathrm{kG}$, similar to other CTTS (Johns-Krull et al. 2003; Valenti \& Johns-Krull 2004), the co-rotation radius of the RW Aur system lies in the range $1.56 R_{\star}<r<2.85 R_{\star}$ (Shu et al. 1994), which is much smaller than the co-rotation radius of a typical T Tauri star $\left(\sim 7-8 R_{\star}\right)$ due to the higher mass accretion rate of RW Aur. Given the uncertainties on the stellar parameters and magnetic field of RW Aur, we set the outer magnetosphere radius to the mean value of $r_{\mathrm{o}}=2.2 R_{\star}$. The inner magnetosphere radius remains a free parameter that was set to $r_{\text {in }}=1.8 R_{\star}$. This magnetosphere width $\left(0.4 R_{\star}\right)$ falls between the narrow $\left(0.2 R_{\star}\right)$ and wide $\left(0.8 R_{\star}\right)$ magnetosphere widths calculated by Muzerolle et al. (2001). A sketch of the magnetosphere and wind geometry is displayed in Fig. 10. 

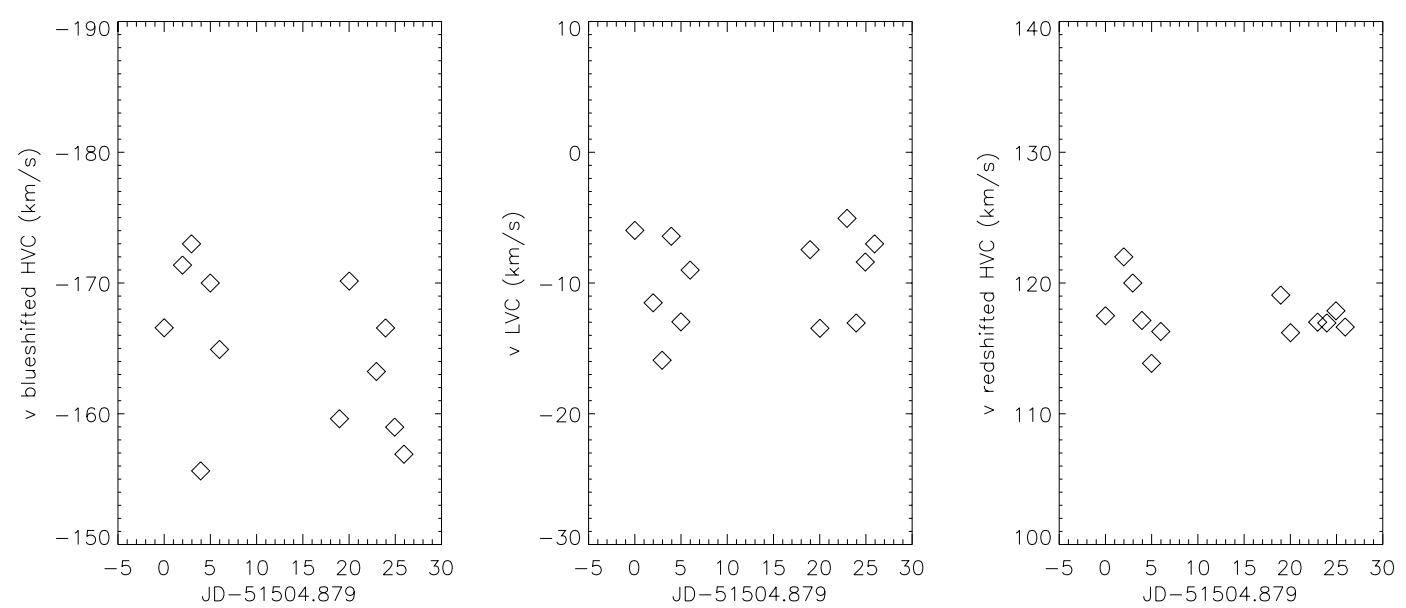

Fig. 8. Central velocity of the $[\mathrm{OI}](6300 \AA)$ line components. The amplitude of the vertical scale in all three plots is $\Delta v=40 \mathrm{~km} \mathrm{~s}^{-1}$.
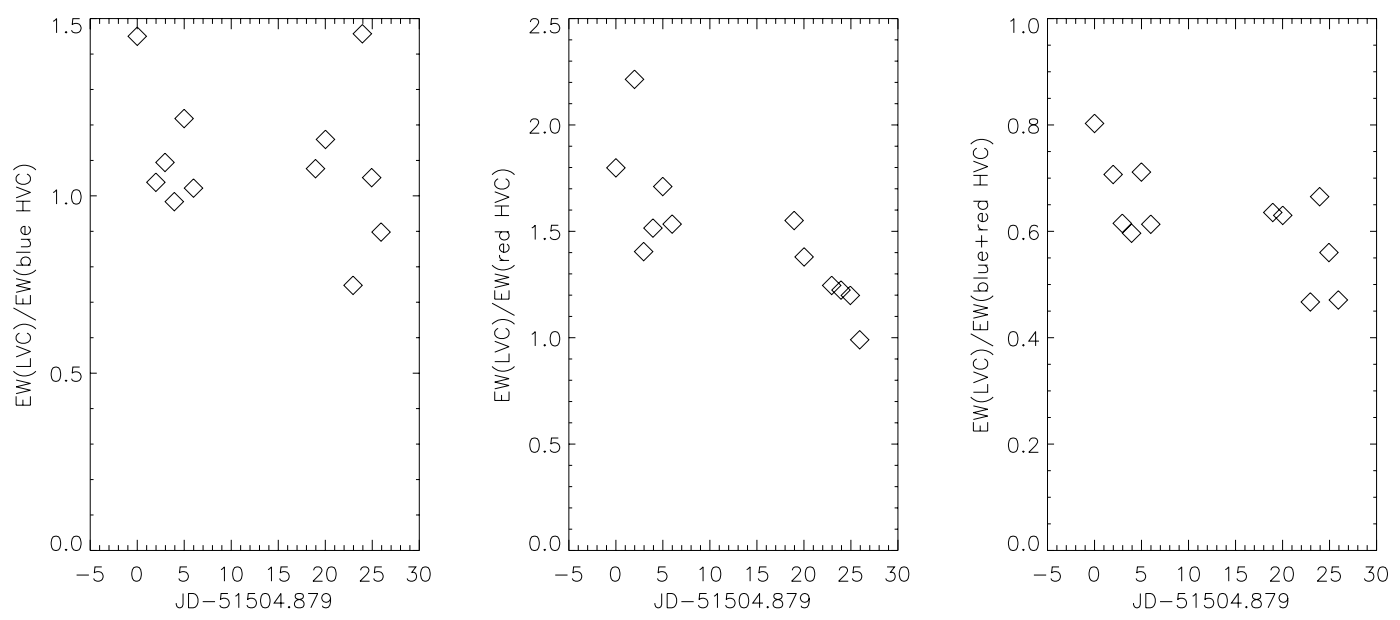

Fig. 9. Equivalent width ratio between the LVC and the HVCs of the $[\mathrm{OI}](6300 \AA$ ) line.

In the left panels of Fig. 12 are shown the mean RW Aur observed $\mathrm{H} \alpha, \mathrm{H} \beta$ and $\mathrm{NaD}$ line profiles and the corresponding magnetospheric accretion profiles overplotted. As in the case of DR Tau (Muzerolle et al. 2001), the magnetospheric accretion profiles of $\mathrm{H} \alpha$ are much less intense and narrower than the observed one. Part of the $\mathrm{H} \beta$ line flux may come from accretion, as suggested by the results of the higher temperature model, but in general the agreement of the magnetospheric accretion model with the observations is rather poor. We must also caution that in order to calculate models with $T_{\max }=7000 \mathrm{~K}$ we had to use a very high heating rate, that, if used in the disk-wind calculations, would generate NaD profiles much more intense than the observed ones. For DR Tau Muzerolle et al. (2001) were able to reproduce the observed $\mathrm{NaD}$ line with the magnetospheric accretion model, except for the narrow blueshifted absorption component that should come from the wind. They argue that the much lower optical depth of the sodium lines precludes emission from the wind but there is enough density at the base of the magnetosphere to generate the observed sodium emission. Appenzeller et al. (2005) however have shown that $\mathrm{NaD}$ can be also produced in the region where we performed our disk-wind calculations. In RW Aur the observed $\mathrm{NaD}$ blueshifted absorption is very deep and wide, indicating that the wind contribution to the line is important. The presence of strong blueshifted emission at $v<-150 \mathrm{~km} \mathrm{~s}^{-1}$ (see Fig. 2) cannot be explained by magnetospheric accretion that tends to generate rather narrow sodium line profiles centered at rest velocity, like in DR Tau. Therefore we argue that in RW Aur a significant fraction of the sodium lines, like the Balmer lines, may come from the wind.

Recently Woitas et al. (2002) traced the RW Aur outflow down to the stellar position in $\mathrm{H} \alpha$ in both lobes. They say there are two $\mathrm{H} \alpha$ velocity peaks on the star with the same velocities as the blue and redshifted lobes of the jet, suggesting they are tracing the jet lobes back to their source. They suggested that the $\mathrm{H} \alpha$ line of RW Aur could be mostly produced in a wind instead of in the magnetosphere.

Following Woitas et al. (2002) suggestion, we tried to investigate how much of the main emission lines of RW Aur could be due to winds. We calculated wind models using the Blandford \& Payne (1982) representation for the wind, which assumes ideal, axisymmetric MHD and steady flow from a Keplerian accretion disk. With the above assumptions, if we specify the poloidal structure of the magnetic field, we can derive the poloidal and toroidal velocity components and the density from mass conservation. We assume the problem is selfsimilar, so the solution for one field line can be scaled for other field lines. Once we have a disk-wind model set, we solve the 


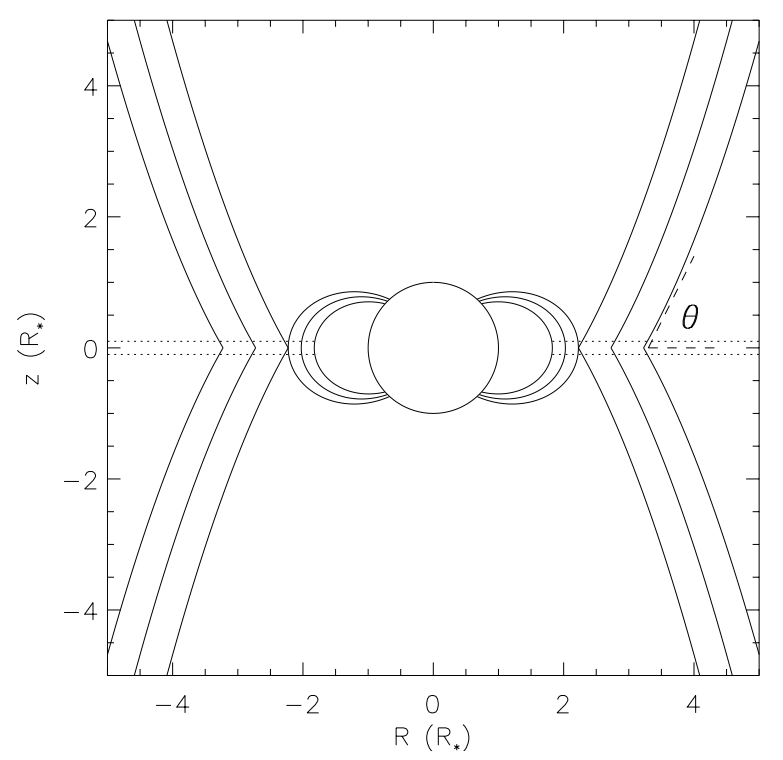

Fig. 10. Sketch of the magnetosphere and wind geometries used in the models. The calculations were extended up to $100 R_{\star}$ in the $z$ direction. The launching angle $(\theta)$ is the angle the wind field line makes with the disk.
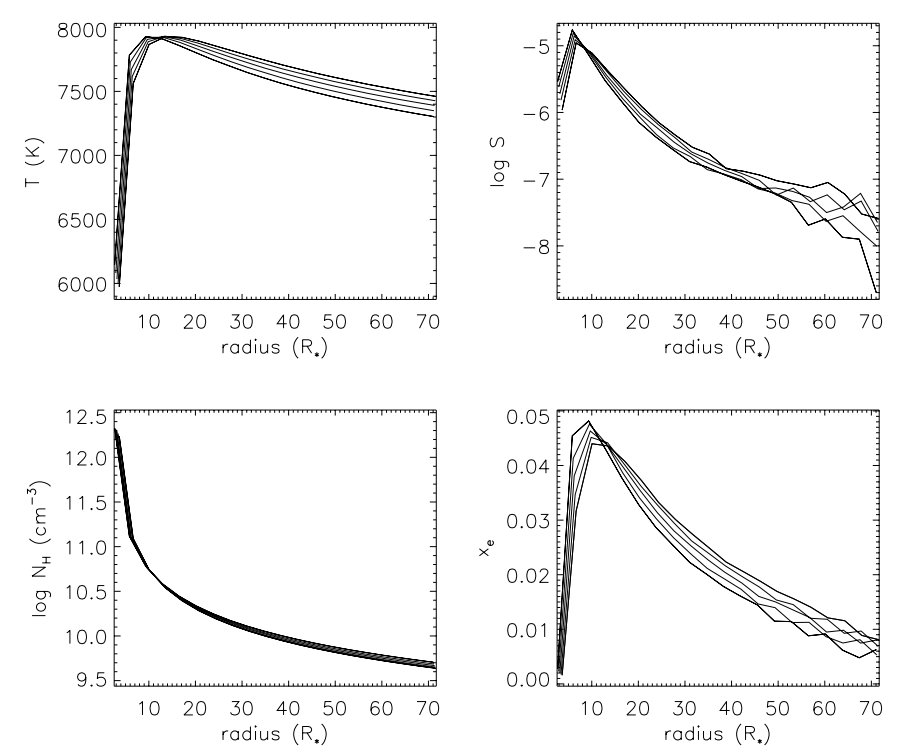

Fig. 11. Temperature, source function, hydrogen density and hydrogen ionization fraction distributions for a model calculated with $M_{\star}=$ $1 M_{\odot}, R_{\star}=2 R_{\odot}, T_{\text {eff }}=4600 \mathrm{~K}, \log \left(\dot{M}_{\mathrm{acc}} / M_{\odot} \mathrm{yr}^{-1}\right)=-6, r_{\mathrm{i}}=2.2 R_{\star}$, $r_{\mathrm{o}}=3.2 R_{\star}$. The lines denote the variation of properties along the disk-wind field lines.

radiative transfer equations using the Sobolev method, following the procedure outlined in Hartmann et al. (1994) for the magnetospheric accretion case. The main difference is that, if the line of sight intersects the disk, we now account for the disk contribution to the emergent line profile. The wind temperature is determined balancing an optically thin radiative cooling rate $\left(N_{\mathrm{H}}^{-2}\right)$ given by Hartmann et al. (1982) with an adjustable heating rate $\left(\propto Q / r^{3}\right)$, such as one would expect if the main heating source were dissipation of magnetic waves. We used a simple two-level plus continuum atom approximation with rotation but no line damping and this will certainly underpredict line widths, but we will still be able to explore the overall importance of winds to the permitted line profiles.

We set the footpoint of the inner wind field line at $2.2 R_{\star}$, starting where the magnetosphere ends. We tested disk-wind configurations with various degrees of wind collimation, $30^{\circ} \leq$ $\theta \leq 60^{\circ}$, where $\theta$ is the launching angle shown in Fig. 10, and different star-disk inclinations $\left(0^{\circ} \leq i \leq 90^{\circ}\right)$. We also calculated disk-wind models extending in the $z$ direction up to $100 R_{\star}$ and with outer wind footpoint radii $\left(r_{\mathrm{o}}\right)$ going from $3.2 R_{\star}$ to $100 R_{\star}$.

The best models that simultaneously match the $\mathrm{H} \alpha, \mathrm{H} \beta$ and $\mathrm{NaD}$ observations correspond to collimated narrow winds. What we mean by a narrow wind is a model that starts from the disk only over a limited range of radii not very far from the co-rotation radius (from $2.2 R_{\star}$ to $3.2 R_{\star}$ in our case). An extended disk-wind spanning a larger range of radii along the disk $\left(r_{\mathrm{i}}=2.2 R_{\star}\right.$ and $\left.r_{\mathrm{o}}>8 R_{\star}\right)$ does not generate emission line profiles that fit simultaneously the $\mathrm{H} \alpha, \mathrm{H} \beta$ and $\mathrm{NaD}$ observed ones. As we increase $r_{\mathrm{o}}$, we also increase the low temperature $(\sim 3000 \mathrm{~K}$ to $5000 \mathrm{~K})$ gas available and the sodium line intensity increases much more rapidly than $\mathrm{H} \alpha$ and $\mathrm{H} \beta$. So in order to fit the $\mathrm{H} \alpha$ and $\mathrm{H} \beta$ lines, we end up with a sodium line that is much more intense than the observed ones. This was valid for disk-wind models with various degrees of collimation. In general, with $r_{\mathrm{o}} \geq 8 R_{\star}$, the $\mathrm{H} \alpha$ and $\mathrm{H} \beta$ fit to the observations was also worse than with $r_{\mathrm{o}}=3.2 R_{\star}$.

To launch the wind, the field line has to make at most an angle of $60^{\circ}$ with the disk (Blandford \& Payne 1982). Since RW Aur has a very collimated microjet (jet opening angle of $3-4^{\circ}$, Dougados et al. 2000) that goes at least down to $14 \mathrm{AU}$ from the star (Woitas et al. 2002), we started the calculations with a very collimated wind model (launching angle $\theta \sim 60^{\circ}$ ). We later tried less collimated wind models with $\theta \sim 50^{\circ}, 40^{\circ}$ and $30^{\circ}$ but the fits to the observed profiles were always worse. In general, as we decrease the launching angle of the wind, the blue emission gets too blue and the red emission peak intensity decreases a lot. It is however still possible to find some reasonable agreement with the observations with $\theta \sim 50^{\circ}$. The calculations in the $z$ direction were extended up to $100 R_{\star}$, but our results were not very sensitive to the the extent of the wind within this limited volume.

The best agreement between observations and wind models was obtained with inclination values in the range $55^{\circ} \leq i \leq$ $65^{\circ}$. According to López-Martín et al. (2003), the jet inclination with respect to the line of sight is $i=46^{\circ} \pm 3^{\circ}$, assuming that knot proper motions trace actual fluid motions and not wave pattern speeds. Woitas et al. (2002) point out that even if the observed tangential velocity is a pattern speed, it is still a lower limit for the actual gas tangential speed, so $i \geq 46^{\circ}$. Our suggested inclination values are therefore consistent, within the uncertainties, with the inferred values for the jet inclination angle, supposing the jet is perpendicular to the disk plane.

The temperature distribution, source function, hydrogen density and hydrogen ionization fraction of a typical disk-wind model are displayed in Fig. 11. The temperatures $(6000 \mathrm{~K}<$ $T<8000 \mathrm{~K})$ and hydrogen ionization fractions $(0.005<$ $\left.x_{\mathrm{e}}<0.05\right)$ are overall in the range expected by observations of 

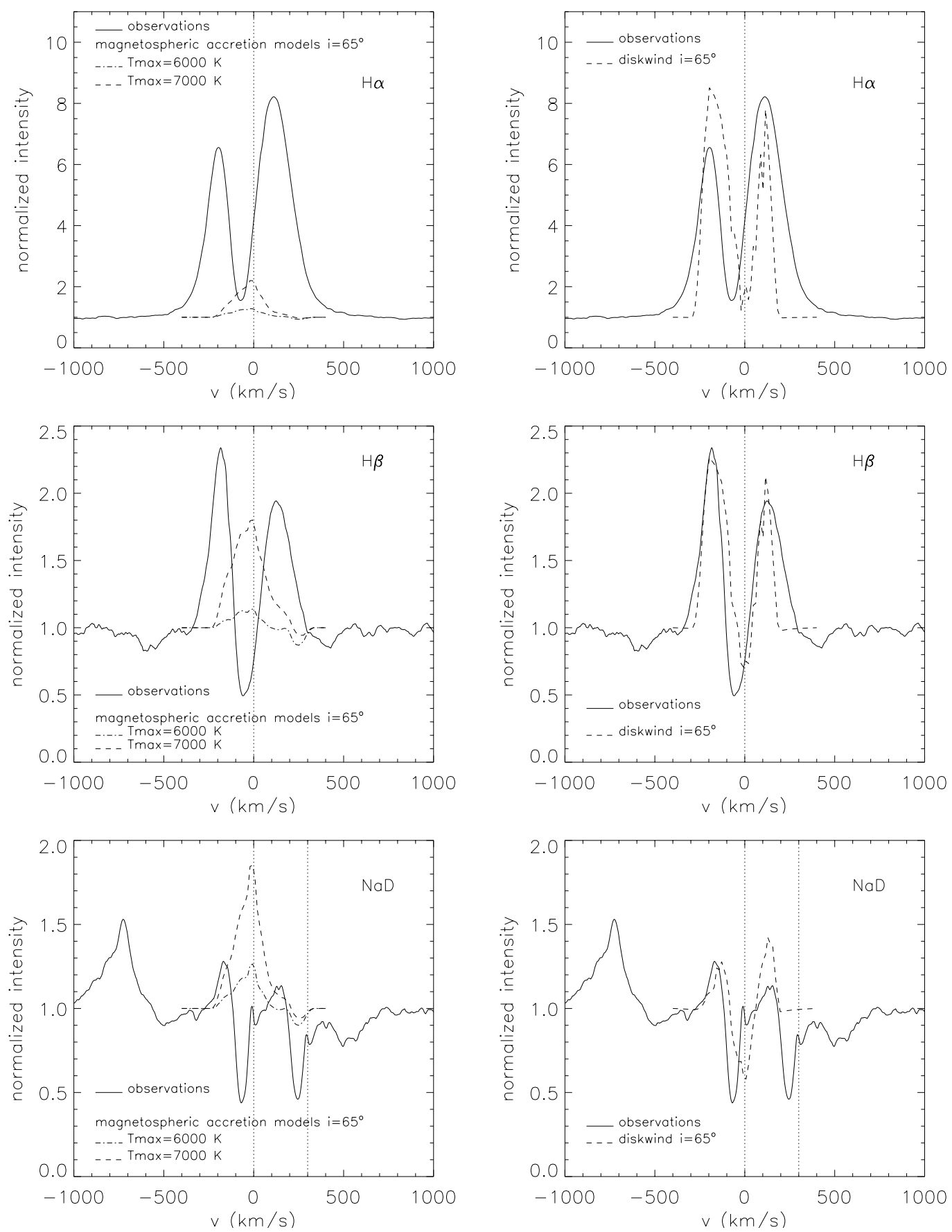

Fig. 12. Mean observed line profiles are shown as solid lines. We did not model the sodium D2 line but only the D1 line. Left: magnetospheric accretion profiles (dashed and dash-dotted lines). Right: disk-wind profiles (dashed lines). Although our wind models are very simple, they provide a much better match to the observed emission line profiles of RW Aur than the magnetospheric accretion models.

optical jets, $T \sim 5000-10000 \mathrm{~K}$ and $x_{\mathrm{e}} \sim 0.01-0.1$ (Bacciotti 2002) and X-wind models (Shang et al. 2002). Our calculation domain is however much smaller than the region observed by Bacciotti (2002) and the large region (0-8000 AU) computed by Shang et al. (2002) and our calculated disk-winds do not reach the higher observed and calculated temperatures $(T>9000 \mathrm{~K})$. Dougados et al. (2002) derived estimates of hydrogen ionization fraction and temperature for RW Aur along the jet in the region $\sim 10 \mathrm{AU}-50 \mathrm{AU}$ away from the star, and thus outside our calculation domain, obtaining for the wind $0.007<x_{\mathrm{e}}<0.09$ and $7000 \mathrm{~K}<T<13500 \mathrm{~K}$. Some of our disk-wind calculated profiles are displayed in the right panels of Fig. 12, overplotted with the mean observed line profiles. There is a general agreement between the disk-wind profiles and the observations, specially taking into account the observed variability of the lines (see Fig. 2) and the simplicity so far of the model (2-level atom, no line damping or turbulence, the Sobolev approximation used to calculate the profiles). Our disk-wind models, however, do not reproduce the outer red emission of the Balmer lines and only part of the 
observed blueshifted absorption is explained by our calculations. This could be mostly due to a computational limitation in the wind extension of our models. Since the line emission was only integrated up to about $100 R_{\star}$, much of the outer cold wind, where most of the blueshifted absorption is thought to arise and part of the receding jet lobe, where some of the far red emission could come from, are not included in the calculations.

We also calculated $\mathrm{H} \alpha, \mathrm{H} \beta$ and $\mathrm{NaD}$ line profiles with a more moderate but still high mass accretion rate $\left(10^{-7} M_{\odot} / \mathrm{yr}\right)$ which is closer to the values obtained by Valenti et al. (1993) and White \& Ghez (2001), using this time the stellar parameters proposed by the latter $\left(M=1.34 M_{\odot}, R=1.7 R_{\odot}\right.$ and $\left.T_{\text {eff }}=5081 \mathrm{~K}\right)$. The main difference is that the co-rotation radius now lies in the range $3.3 R_{\star}<r<4.6 R_{\star}$ and we adopted the mean value of $r_{\mathrm{o}}=4.0 R_{\star}$. The magnetospheric and diskwind profiles obtained are very similar to the ones calculated with the higher mass accretion rate. Once again the magnetospheric accretion profiles do not agree with the observations and we only obtained a good agreement with narrow collimated disk-wind models, now starting from $4 R_{\star}$ to $5 R_{\star}$ along the disk.

Despite the model limitations, comparing the magnetospheric and disk-wind results with the observations (Fig. 12) we see that, in the RW Aur case, the wind contribution to the line profiles is very important and must be taken into account in order to reproduce the observations. It is clear that the correct model to describe CTTSs should actually include both the magnetosphere and an extended disk-wind calculated consistently. This however is beyond the scope of the present work and will be left for a future implementation.

\section{Discussion and conclusions}

The analysis of the correlation matrices of the main emission lines of RW Aur point to a common origin for the $\mathrm{H} \alpha, \mathrm{H} \beta$,

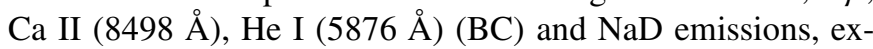
cept for the outer red wings of $\mathrm{H} \alpha, \mathrm{H} \beta$ and Ca II (8498 $\AA$ ) that do not correlate with the rest of the profiles. We have shown in the previous section that disk-wind models can reproduce most of the observed characteristics of the Balmer and $\mathrm{NaD}$ line regions that correlate with each other, suggesting that the wind is a common formation region of the main emission lines observed in RW Aur. This result agrees with the scenario proposed by Beristain et al. (2001) where the He I (5876 ̊) BC blue emission is also mostly due to winds.

However, part of the red emission peak of $\mathrm{H} \alpha$ and $\mathrm{H} \beta$ does not seem to originate either in the magnetosphere or in the wind close to the star $\left(d<100 R_{\star} \sim 1 \mathrm{AU}\right.$, the limit of our calculations). This is evident comparing the observed and computed profiles and also clear from the $\mathrm{H} \alpha$ correlation matrices (Sect. 3.3), since the $\mathrm{H} \alpha$ far red wing $\left(v>200 \mathrm{~km} \mathrm{~s}^{-1}\right)$ does not correlate with the rest of the profile. One possibility is that our observed $\mathrm{H} \alpha$ line has a significant contribution from the red lobe of the jet that was observed closer than $1^{\prime \prime}$ from the star by López-Martín et al. (2003) and Woitas et al. (2002) one year before and one year after our observations respectively. The receding jet is always in sight and that could explain the small variability of the outer red emission. Another possibility to explain the outer wings of the Balmer lines is a significant contribution due to line damping that is certainly important at these high mass accretion rates and that we are not taking into account in the current disk-wind models. The addition of damping might also help to explain why the red wings are not well correlated with other variabilities, since they will naturally be more stable than other regions affected by Doppler shifts.

Woitas et al. (2002) proposed that the observed emission of the RW Aur $\mathrm{H} \alpha$ line can mostly originate in the blue and red jet lobes, since they trace the $\mathrm{H} \alpha$ emission in both lobes back to the stellar position, and the $\mathrm{H} \alpha$ blue and red velocity peaks observed on the star present the same velocities as the blue and redshifted lobes of the jet. The $\mathrm{H} \alpha$ profile is indeed expected to have a strong contribution from the wind, as we have shown in the previous section, but the observed profile cannot mostly come from the outer collimated wind regions since there is evidence that most of the observed $\mathrm{H} \alpha$ line comes from a region that is common to all the broad emission lines, including $\mathrm{NaD}, \mathrm{Ca}$ II $(8498 \AA)$ and the He I (5876 ̊) BC, that are not expected to be produced in the low density outer wind.

Woitas et al. (2002) traced the redshifted jet down to approximately $15 \mathrm{AU}$ from the star in the forbidden emission lines, which ends up being an upper limit for the projected radius of an opaque disk around RW Aur. Although the RW Aur system apparently presents a small accretion disk $\left(R_{\text {disk }} \leq 35\right.$ AU for $i \leq 65^{\circ}$ and a projected disk radius of $15 \mathrm{AU}$ ) compared to standard values commonly attributed to a typical CTTS $\left(R_{\text {disk }} \sim 100 \mathrm{AU}\right)$, the red jet lobe close to the star should still be occulted by the outer projected disk radius at the inclinations suggested by the microjet and our works $\left(43^{\circ} \leq i \leq 65^{\circ}\right)$. The receding jet should not be visible either through the inner disk gap. Due to the high mass accretion rate of the system, the co-rotation radius lies very close to the star $\left(r_{\text {co }} \sim 2.2 R_{\star}\right)$ and the receding inner jet is therefore almost totally occulted by the star and the inner disk edge at the inclinations corresponding to the best model fits. What causes the double-peaked profile in our models is a geometrical effect of the velocity projection of the material in the open wind field lines. The disk-wind actually behaves as a series of expanding and rotating rings that naturally gives rise to a double-peaked line profile.

Woitas et al. (2005) recently measured the rotation of the RW Aur bipolar jet using STIS-HST. They estimated that the wind detected in the optical forbidden lines is launched within about $0.5 \mathrm{AU}$ from the star for the blue lobe and within 1.6 $\mathrm{AU}$ for the red lobe, thus predicting a disk-wind that extends over several stellar radii, which is different from what we found in this work. They also obtained a magnetic lever arm (the ratio between the Alfvén and the footpoint radii) in the range $3.5 \leq r_{\mathrm{A}} / r_{0} \leq 4.6$, which is in agreement with the value used in our models $\left(4.2 \leq r_{\mathrm{A}} / r_{0} \leq 4.5\right)$ and falls within the values predicted by the Casse \& Ferreira (2000) and Ferreira (2002) wind models, being actually consistent with their warm solutions and thus suggesting that some heating must be provided at the base of the jet. It would be very interesting to calculate the Balmer and Sodium line emissions predicted by extended warm wind solutions to see if they are consistent with our observations, in the sense that the wind contribution to the permitted line emissions cannot exceed the observed ones. 
Basri \& Batalha (1990) analyzed some of the $3 \mathrm{~m}$ spectra used in this study and saw no correlation between the $\mathrm{H} \alpha$ equivalent width and veiling but found a strong correlation between the $\mathrm{H} \alpha$ flux and veiling in RW Aur. This is what is expected if the power in the line increased proportionally to the continuum. In the context we propose here, where most of the $\mathrm{H} \alpha$ line of RW Aur arises in the wind, this correlation result supports the idea of a direct connection between the accretion and outflow processes.

We saw strong evidence with our data that the outflow process is highly dynamic. For example, the [OI](6300 $\AA$ ) forbidden line, which is supposed to be formed in the disk-wind (LVC) and the jet (HVC), was observed to vary on a timescale of hours in our observations. The apparent disappearance of the LVC from December 1999 to December 2000 shows that the wind can present a dramatic variability on longer timescales too.

We also saw evidence from the periodogram analysis of the main emission lines that the hot spot configuration may have changed in a period of a few years, implying a significant change in the magnetospheric configuration of the system in such a short timescale. The dynamic nature of the inflow/outflow process in CTTSs on several timescales was also observed by Bouvier et al. (2003) analyzing synoptic observations of the CTTS AA Tau.

Overall we have seen that the contribution of the disk-wind to the observed line profiles of this extreme CTTS is very significant and must be taken into account in order to describe the spectroscopic observations. The flux in the disk-wind of RW Aur is particularly intense and about the same order of magnitude as the jet flux. Accretion is certainly occurring at the same time, but due to the characteristics of the system (high mass accretion rate, small magnetosphere and consequently high densities, low temperatures and small emitting area in the magnetosphere), the influence of magnetospheric accretion on the observed emission line profiles is not very strong.

Our results show the need for time variable CTTS models that include both the magnetosphere and the disk-wind calculated in a consistent way in order to describe the synoptic observations of such active young stars.

Acknowledgements. This research is based on data collected on the Shane $3 \mathrm{~m}$ and CAT telescopes at Lick Observatory run by the University of California. S.H.P.A. acknowledges support from CAPES (PRODOC program) and CNPq (grant 201228/2004-1). G.B. acknowledges support from the NSF through grant AST86-16863. N.C. and L.H. acknowledge support from NASA through grant NAG513210 and NASA grant GO-08627.01-A from the Space Telescope Science Institute.

\section{References}

Alencar, S. H. P., \& Basri, G. 2000, AJ, 119, 1881

Appenzeller, I., Bertout, C., \& Stahl, O. 2005, A\&A, accepted Bacciotti, F. 2002, Rev. Mex. Astron. Astrofis. Conf. Ser., 13, 8 Basri, G., \& Batalha, C. 1990, ApJ, 363, 654

Basri, G., Marcy, G., \& Valenti, J. 1992, ApJ, 390, 622
Basri, G., Johns-Krull, C. M., \& Mathieu, R. D. 1997, AJ, 114, 781

Beristain, G., Edwards, S., \& Kwan, J. 2001, ApJ, 551, 1037

Bertout, C., Basri, G., \& Bouvier, J. 1988, ApJ, 330, 350

Bertout, C. 1989, ARA\&A, 27, 351

Blandford, R. D., \& Payne, D. G. 1982, MNRAS, 199, 883

Bouvier, J., Grankin, K. N., Alencar, S. H. P., et al. 2003, A\&A, 409, 169

Calvet, N., \& Gullbring, E. 1998, ApJ, 509, 802

Casse, F., \& Ferreira, J. 2000, A\&A, 353, 1115

Coffey, D., Bacciotti, F., Woitas, J., Ray, T. P., \& Eislöffel, J. 2004, ApJ, 604, 758

Dougados, C., Cabrit, S., Lavalley, C., \& Ménard, F. 2000, A\&A, 357, L61

Dougados, C., Cabrit, S., \& Lavalley-Fouquet, C. 2002, Rev. Mex. Astron. Astrofis. Conf. Ser., 13, 43

Ferreira, J. 2002, Proc. Star Formation and the Physics of Young Stars, ed. J. Bouvier, \& J.-P. Zahn (EDP Sciences), 3, 229

Gahm, G. F., Petrov, P. P., Duemmler, R., Gameiro, J. F., \& Lago, M. T. V. T. 1999, A\&A, 352, L95

Ghez, A. M., Neugebauer, G., \& Matthews, K. 1993, AJ, 106, 2005

Goodson, A. P., Böhm, K., \& Winglee, R. M. 1999, ApJ, 524, 142

Hartigan, P., Edwards S., \& Ghandour, L. 1995, ApJ, 452, 736

Hartmann L., Edwards S., \& Avrett E. 1982, ApJ, 261, 279

Hartmann L., Calvet, N., Avrett E., \& Loesler R. 1990, ApJ, 349, 168

Hartmann, L., Hewett, R., \& Calvet, N. 1994, ApJ, 426, 669

Hirth, G. A., Mundt, R., \& Solf, J. 1997, A\&AS, 126, 437

Johns, C. M., \& Basri, G. 1995a, AJ, 109, 2800

Johns, C. M., \& Basri, G. 1995b, ApJ, 449, 341

Johns-Krull, C. M., Valenti, J. A., \& Gafford, A. D. 2003, Rev. Mex. Astron. Astrofis. Conf. Ser., 18, 38

Kuhi, L.V. 1964, ApJ, 140, 1409

Kwan, J., \& Tademaru, E. 1988, ApJ, 332, L41

Kwan, J., \& Tademaru, E. 1995, ApJ, 454, 382

López-Martín, L., Cabrit, S., \& Dougados, C. 2003, A\&A, 405, L1

Muzerolle J., Hartmann L., \& Calvet N. 1998, AJ, 116, 455

Muzerolle J., Hartmann L., \& Calvet N. 2001, ApJ, 550, 944

Natta A., \& Giovanardi C. 1990, ApJ, 356, 646

Pesenti, N., Dougados, C., Cabrit, S., et al. 2003, A\&A, 410, 155

Petrov, P. P., Gahm, G. F., Gameiro, J. F., et al. 2001, A\&A, 369, 993

Romanova, M. M., Ustyugova, G. V., Koldoba, A. V., Wick, J. V., \& Lovelace, R. V. E. 2003, ApJ, 595, 1009

Shang, H., Glassgold, A. E., Shu, F. H., \& Lizano, S. 2002, ApJ, 564, 853

Shu, F., Najita, J., Ostriker, E., et al. 1994, ApJ, 429, 781

Solf, J. 1997, Herbig-Haro Flows and the Birth of Stars, ed. Bo Reipurth, \& C. Bertout (Kluwer Academic Publishers), IAU Symp., 182, 63

Stout-Batalha, N. M., Batalha, C. C., \& Basri, G. S. 2000, ApJ, 532, 474

Symington, N. H., Harries, T. J., \& Kurosawa, R. 2005, MNRAS, 356, 1489

Valenti, J. A., Basri, G., \& Johns, C. M. 1993, AJ, 106, 2024

Valenti, J. A. 1994, Ph.D. Thesis, Univ. of California, Berkeley

Valenti, J. A., \& Johns-Krull, C. M. 2004, Ap\&SS, 292, 619

Vogt, S.S. 1987, PASP, 99, 1214

White, R. J., \& Ghez, A. M. 2001, ApJ, 556, 265

Woitas, J., Ray, T. P., Bacciotti, F., Davis, C. J., \& Eislöffel, J. 2002, ApJ, 580, 336

Woitas, J., Ray, T. P., Bacciotti, F., Davis, C. J., \& Eislöffel, J. 2005, A\&A, accepted 